\title{
MicroReview
}

\section{Differentiate to thrive: lessons from the Legionella pneumophila life cycle}

\author{
Ari B. Molofsky and Michele S. Swanson* \\ Department of Microbiology and Immunology, University \\ of Michigan Medical School, 6734 Medical Sciences \\ Building II, Ann Arbor, MI 48109-0620, USA.
}

\section{Summary}

When confronted by disparate environments, microbes routinely alter their physiology to tolerate or exploit local conditions. But some circumstances require more drastic remodelling of the bacterial cell, as sporulation by the Bacillus and Streptomyces species of soil bacteria vividly illustrates. Cellular differentiation is also crucial for pathogens, the challenge for which is to colonize one host, then be transmitted to the next. Using the Gram-negative Legionella pneumophila as a model intracellular pathogen, we describe how biogenesis of the replication vacuole is determined by the developmental state of the bacterium. Subsequently, when replicating bacteria have exhausted the nutrient supply, the pathogens couple their conversion to stationary phase physiology with expression of traits that promote transmission to a new host. The cellular differentiation of $L$. pneumophila is co-ordinated by a regulatory circuit that integrates several elements that are broadly conserved in the microbial world. The alarmone (p)ppGpp promotes transcription directed by the alternative sigma factors RpoS, FliA and, probably, RpoN, and also post-transcriptional control mediated by a two-component regulatory system, LetA/S (GacA/S), and an mRNA-binding protein, CsrA (RsmA). By applying knowledge of microbial differentiation in combination with tools to screen the complete genomes of pathogens, experiments can be designed to identify two distinct classes of virulence traits: factors that promote replication and those dedicated to transmission.

Accepted 15 March, 2004. *For correspondence. E-mail mswanson@umich.edu; Tel. (+1) 734647 7295; Fax (+1) 734764 3562.

(C) 2004 Blackwell Publishing Ltd

\section{'Adapt yourself to changing circumstances'}

Even the simplest of organisms heed this Chinese proverb, as bacteria acclimate to fluctuations in their environments. Whether to make use of local energy sources or to tolerate a range of acidity, osmolarity or temperature, microbes adjust their physiology via sophisticated signal transduction pathways. Unicellular organisms can also respond to environmental cues by activating morphogenetic programmes during a life cycle, a commitment that we shall distinguish from metabolic regulation by referring to it as differentiation.

Conspicuous examples of microbial differentiation are the obligate intracellular pathogens, which must periodically abandon a cytoplasmic or vacuolar niche to face unpredictable circumstances during their transmission to a new host. For example, Coxiella burnetii, which causes $Q$ fever, alternates between the replicative large cell variant and the environmental small cell variant, a form that is highly stress resistant (reviewed by Samuel et al., 2003). Similarly, during its biphasic life cycle, the common sexually transmitted pathogen Chlamydia trachomatis changes between an intracellular replicative reticulate body and a resilient and infectious elementary body (reviewed by Hammerschlag, 2002). Because neither pathogen can be manipulated genetically, identification of the regulatory circuits that control their life cycles is a formidable challenge.

Insight into the molecular mechanisms of differentiation by pathogens has been obtained from studies of more tractable Gram-negative bacteria. The Bordetella species are extracellular respiratory pathogens that use a BvgA/S phosphorelay together with sigma factors that regulate both motility (Frl) and a type III secretion system (BtrS) to control expression of three sets of traits: those thought to promote colonization of the respiratory tract (Bvg form), transmission to a new host (Bvg' form) and survival in the environment (Bvg- form; reviewed by Cotter and Jones, 2003; S. Matoo and J. F. Miller, personal communication). As an experimental model for analysing a pathogen's reciprocal expression of replicative and transmissive traits and its impact on host cell biology, we focus here on the Gram-negative intracellular bacterium Legionella pneumophila. 


\section{Emergence from amoebae to human pathogen}

Ubiquitous in aquatic environments, L. pneumophila endures a range of temperatures, osmolarity and other stresses, including ingestion by protozoa that feed on bacteria. Aerosolized microbes that are inhaled by humans can also replicate inside alveolar macrophages. When a robust host defence is absent, the progressive and potentially fatal pneumonia known as Legionnaires' disease develops (reviewed by Fields et al., 2002; Swanson and Hammer, 2000). However, as L. pneumophila do not spread from person to person, humans have been inconsequential to the evolution of its virulence. Rather, the ability of $L$. pneumophila to colonize alveolar macrophages probably reflects conservation of cell biological pathways between the professional phagocytes that patrol the lungs and those that inhabit ponds. Indeed, the microbe's life cycle in amoebae and macrophages is remarkably similar (Fields et al., 2002), bolstering the notion that the virulence strategy of $L$. pneumophila has been shaped by selective pressures in aquatic ecosystems.

\section{Cycles of replication and transmission}

Legionella differentiation

In natural and potable fresh water supplies, L. pneumophila probably resides within biofilm communities, where it falls prey to grazing amoebae. When ingested, the microbe can resist digestion and, instead, replicates profusely before killing its protozoan host and returning to the aquatic reservoir. As predicted for a microbe that transits between phagocytes and water, the $L$. pneumophila life cycle consists of at least two phases. In pioneering studies, Rowbotham (1986) infected amoebae and watched as the bacteria alternated between two morphologically distinct forms, changing motility, shape, surface and stores of energy-rich polymers. Subsequent analysis of $L$. pneumophila differentiation has been expedited by the discovery that many aspects of the pathogen's life cycle can be modelled in synchronous broth cultures, as judged by the growth phase-dependent expression of numerous traits and genes (Table 1). Identification of regulatory elements that govern the bacterial life cycle has also been advanced by the development of genetic tools to study $L$. pneumophila, including transposon mutagenesis, transformation via natural competence, conjugation and electroporation, recombinant green fluorescent protein $(g f p)$ genes and the genome sequence. Accordingly, the working model put forward here assimilates data obtained from several independent morphological, genetic and molecular approaches.

In the model's simplest form, the L. pneumophila life cycle is composed of two reciprocal phases: replication and transmission (Fig. 1). When conditions are favourable

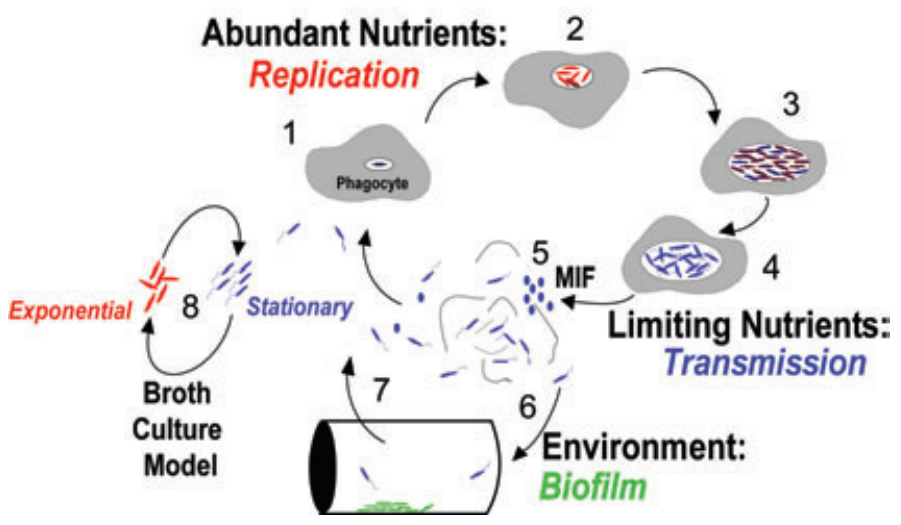

Fig. 1. The life cycle of $L$. pneumophila. Studies of broth and phagocyte laboratory cultures support the following model for the persistence of $L$. pneumophila in aquatic reservoirs.

1. Free-swimming transmissive L. pneumophila that are engulfed by phagocytic cells (amoebae or alveolar macrophages) establish vacuoles that provide protection from lysosomal digestion.

2. When nutrients and other conditions are favourable, intracellular bacteria repress transmission traits and activate pathways that promote replication.

3. As conditions in the replication compartment deteriorate, the progeny stop dividing and co-ordinately express traits that promote survival in the environment and transmission to a new phagocytic host.

4. After a prolonged period, the microbes may continue to develop into a mature intracellular form (MIF), a cell type that is highly resilient and infectious.

5. The phagocyte host is lysed, and the microbes are released into the aqueous environment.

6. L. pneumophila that do not immediately encounter a new phagocyte probably establish biofilms in both water systems and ponds, where they are resistant to biocidal agents.

7. When planktonic microbes encounter a new phagocyte, the cycle begins anew.

8. Microbes cultured in broth to either exponential or stationary phase display many attributes of the replicative and transmissive forms, respectively, that are observed in phagocyte cultures. 
Table 1. Reciprocal expression of multiple traits by replicative and transmissive L. pneumophila.

\begin{tabular}{|c|c|c|c|c|}
\hline Traits & $\begin{array}{l}\text { Replicative } \\
\text { phase }\end{array}$ & $\begin{array}{l}\text { Transmissive } \\
\text { phase }\end{array}$ & Regulators & References \\
\hline Replication ${ }^{\star a}$ & + & - & +: CsrA, RpoS? & $\begin{array}{l}\text { Bachman and Swanson (2001); } \\
\text { Molofsky and Swanson (2003); } \\
\text { Bachman and Swanson (2004a) }\end{array}$ \\
\hline $\begin{array}{l}\text { CsrA global repressor } \\
\text { expression }^{\star a}\end{array}$ & + & - & -: LetA/S & $\begin{array}{l}\text { Fettes et al. (2001); } \\
\text { Molofsky and Swanson (2003) }\end{array}$ \\
\hline $\begin{array}{l}\text { (p)ppGpp } \\
\text { accumulation }\end{array}$ & - & + & +: RelA, SpoT? & $\begin{array}{l}\text { Hammer and Swanson (1999); } \\
\text { Zusman et al. (2002) }\end{array}$ \\
\hline $\begin{array}{l}\text { Motility/ } \\
\text { flagellar } \\
\text { regulon*a,b }\end{array}$ & - & + & $\begin{array}{l}\text { +: RelA, LetA/S, } \\
\text { LetE, RpoS, } \\
\text { RpoN/FleQ, FliA } \\
\text {-: CsrA, FlaR, } \\
\text { RpoS }\end{array}$ & $\begin{array}{l}\text { Byrne and Swanson (1998); } \\
\text { Fettes et al. (2001); } \\
\text { Hammer et al. (2002); } \\
\text { Heuner et al. (1999); } \\
\text { Heuner et al. (2000); } \\
\text { Lynch et al. (2003); } \\
\text { Molofsky and Swanson (2003); } \\
\text { Rowbotham (1986); } \\
\text { Zusman et al. (2002); } \\
\text { Bachman and Swanson (2004a,b); } \\
\text { Jacobi et al. (2004) }\end{array}$ \\
\hline $\begin{array}{l}\text { Contact-dependent } \\
\text { cytotoxicity }{ }^{\star a}\end{array}$ & - & + & $\begin{array}{l}\text { +: LetA/S, FliA } \\
\text {-: CsrA }\end{array}$ & $\begin{array}{l}\text { Alli et al. (2000); } \\
\text { Byrne and Swanson (1998); } \\
\text { Hammer et al. (2002); } \\
\text { Molofsky and Swanson (2003) }\end{array}$ \\
\hline Stress resistance & - & + & $\begin{array}{l}\text { +: LetA/S, LetE } \\
\text {-: CsrA }\end{array}$ & $\begin{array}{l}\text { Bachman and Swanson (2001); } \\
\text { Hales and Shuman (1999); } \\
\text { Lynch et al. (2003); } \\
\text { Molofsky and Swanson (2003); } \\
\text { Bachman and Swanson (2004b) }\end{array}$ \\
\hline Pigment production & - & + & $\begin{array}{l}\text { +: LetA/S, LetE, } \\
\text { RelA } \\
\text {-: CsrA }\end{array}$ & $\begin{array}{l}\text { Molofsky and Swanson (2003); } \\
\text { Zusman et al. (2002); } \\
\text { Bachman and Swanson (2004b); } \\
\text { Fettes et al. (2001) }\end{array}$ \\
\hline $\begin{array}{l}\text { Beta-hydroxybutyrate } \\
\text { storage granules }{ }^{\star b, c}\end{array}$ & - & + & ND & $\begin{array}{l}\text { Faulkner and Garduno (2002); } \\
\text { Greub and Raoult (2003); } \\
\text { Rowbotham (1986) }\end{array}$ \\
\hline $\begin{array}{l}\text { Small coccoid } \\
\text { cells }\end{array}$ & - & + & $\begin{array}{l}\text { +: LetA/S, LetE } \\
\text {-: CsrA }\end{array}$ & $\begin{array}{l}\text { Faulkner and Garduno (2002); } \\
\text { Fettes et al. (2001); } \\
\text { Molofsky and Swanson (2003); } \\
\text { Rowbotham (1986); } \\
\text { Bachman and Swanson (2004b) }\end{array}$ \\
\hline Lysosome evasion*a & - & + & $\begin{array}{l}\text { +: LetA/S, FliA } \\
\text {-: CsrA }\end{array}$ & $\begin{array}{l}\text { Byrne and Swanson (1998); } \\
\text { Hammer et al. (2002); } \\
\text { Joshi et al. (2001); } \\
\text { Molofsky and Swanson (2003); } \\
\text { Sturgill-Koszycki and Swanson (2000) }\end{array}$ \\
\hline $\begin{array}{l}\text { Endoplasmic } \\
\text { reticulum } \\
\text { recruitment }^{\star a}\end{array}$ & - & + & ND & $\begin{array}{l}\text { A. O. Amer and M. Swanson (in } \\
\text { preparation) }\end{array}$ \\
\hline Sodium sensitivity*a & - & + & $\begin{array}{l}\text { +: LetA/S, LetE, } \\
\text { RpoS } \\
-: \text { CsrA }\end{array}$ & $\begin{array}{l}\text { Bachman and Swanson (2001); } \\
\text { Byrne and Swanson (1998); } \\
\text { Hammer et al. (2002); } \\
\text { Molofsky and Swanson (2003); } \\
\text { Bachman and Swanson (2004b) }\end{array}$ \\
\hline $\begin{array}{l}\text { RalF, LidA }{ }^{d} \text { or } \\
\text { SidC expression - } \\
\text { Dot/lcm substrates }\end{array}$ & Low & High & ND & $\begin{array}{l}\text { Conover et al. (2003); } \\
\text { Luo and Isberg }(2004) \\
\text { Nagai et al. }(2002)\end{array}$ \\
\hline $\begin{array}{l}\text { Mip }^{d}, \text { DotO }^{d}, \text { DotH }^{d} \\
\text { MagA }^{c} \text { expression - }\end{array}$ & Low & High & $+: \operatorname{RpoS}(\mathrm{Mag} A)$ & $\begin{array}{l}\text { Watarai et al. (2001a); } \\
\text { Wieland et al. (2002); } \\
\text { Bachman and Swanson (2004a); } \\
\text { Hiltz et al. (2004) }\end{array}$ \\
\hline
\end{tabular}

Traits regulated similarly by L. pneumophila cultured in both broth and macrophages ${ }^{\mathrm{a}}$, amoebae ${ }^{\mathrm{b}}$ or HeLa cells ${ }^{\mathrm{c}}$ are indicated by ${ }^{*}$. Traits only regulated during macrophage infection but not in broth culture are indicated with ${ }^{\mathrm{d}}$. Regulators controlling various traits are indicated: - indicates repression; + indicates activation; ND indicates that experiments to identify regulators have not been done. 
for replication, traits that promote transmission are repressed, and the intracellular bacteria multiply (Fig. 2A). As vacuolar nutrients become limiting, the progeny differentiate into the transmissive phase, repressing multiplication while expressing a number of traits that are believed to equip L. pneumophila to escape from its spent host cell, survive as a planktonic cell and re-establish a replicative niche within a new phagocyte (Fig. 2B). In particular, as broth cultures enter the stationary phase, the bacteria coordinately express: (i) an inducer of phagocyte necrosis; (ii) motility; (iii) resistance to the stresses of UV light, heat, osmotic pressure and nutrient limitation; and (iv) factors to evade degradation within phagocyte lysosomes (Table 1 and references therein). We define these phenotypes as transmission traits to emphasize that none is expressed by replicating bacteria and all are thought to promote spread of the pathogen from its protective vacuole in one host to that in another. After successfully assembling another intracellular niche, L. pneumophila reverts to the replicative form, beginning the cycle anew.
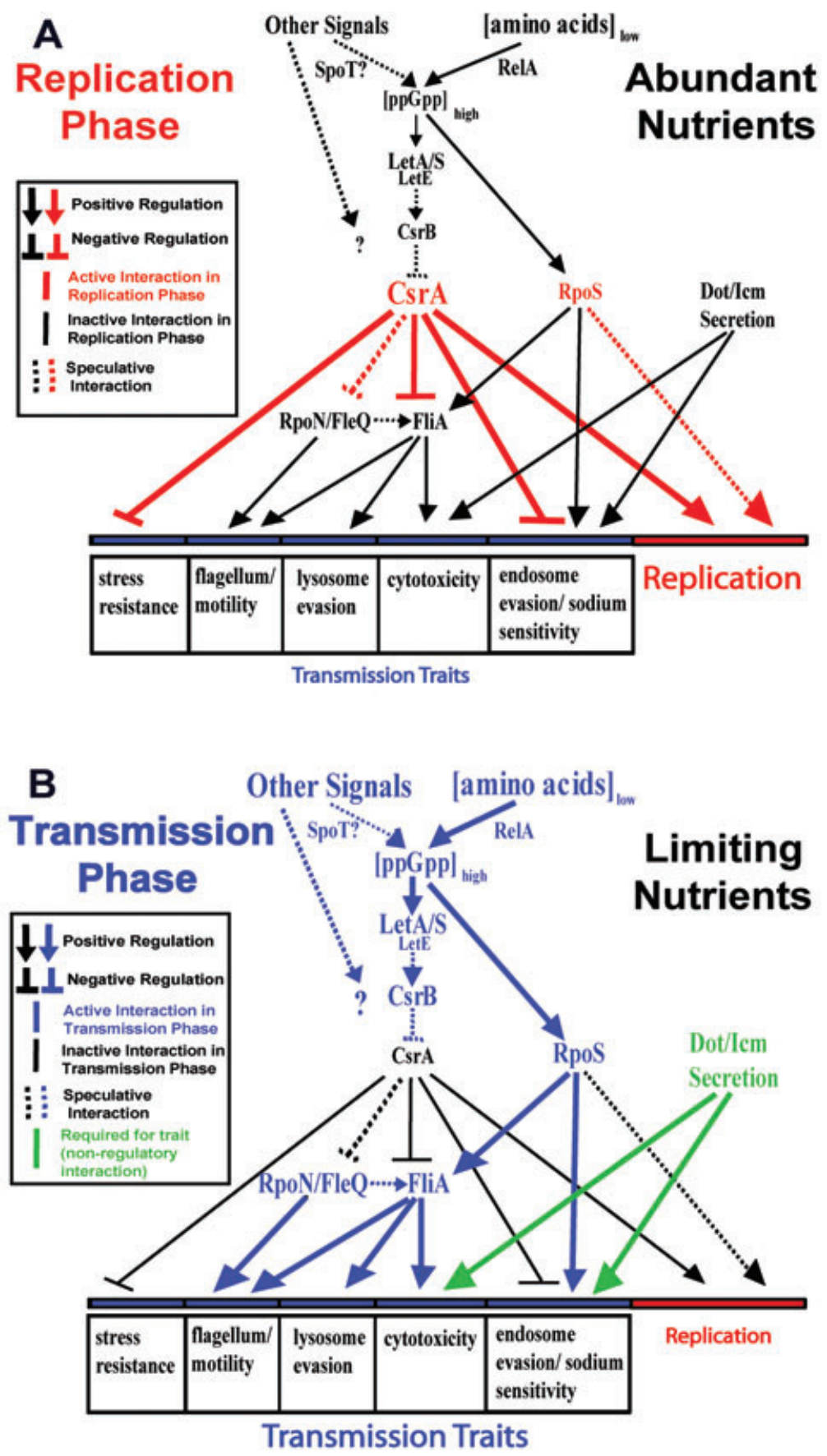

Fig. 2. Reciprocal expression of the replicative and transmissive phenotypes is co-ordinated by (p)ppGpp levels and an integrated network of alternative sigma factors and a posttranscriptional control regulatory system. A model deduced from genetic studies of regulatory interactions that may be direct or indirect is shown.

A. During the replicative phase, nutrients are abundant, and the post-transcriptional regulator CsrA represses transmission traits and promotes replication. The alternative sigma factor RpoS also stimulates intracellular replication and osmotic resistance and inhibits FliA-dependent traits. CsrA inhibits flagellar production and the co-regulated traits of lysosomal evasion and induction of phagocyte necrosis (cytotoxicity) by repressing the flagellar sigma factor FliA $\left(\sigma^{28}\right)$ directly or indirectly or perhaps by regulating the putative master flagellar regulators $\operatorname{RpoN}\left(\sigma^{54}\right)$ and/or FleQ. CsrA also represses resistance to several environmental stresses, sodium sensitivity and endosomal evasion. B. During the transmissive phase, a poor amino acid supply may trigger the ribosomalassociated enzyme RelA to produce the alarmone (p)ppGpp. Alternative intracellular signals may stimulate the SpoT hydrolase/synthase to produce the alarmone. (p)ppGpp stimulates the two-component system LetA/S, the enhancer protein LetE, the alternative sigma factor RpoS and perhaps the putative master flagellar regulator RpoN, which together promote the expression of transmission traits. Active LetA may induce the production of a $\operatorname{csr} B$-like regulatory RNA to sequester CsrA and relieve CsrA posttranscriptional repression, whereas RpoS, FliA and RpoN probably activate transcription of the transmission regulon genes. In macrophage culture, undefined signal(s) can bypass both RelA and LetA to induce transmission. FliAdependent and -independent pathways are induced that promote escape, motility, resilience and infection of a new phagocyte host. Type IV Dot/lcm secretion is required for cytotoxicity as well as evasion of the endocytic network, probably by directly exporting the effectors of virulence, whereas Rpos induces expression of the FliA regulon and traits that confer endosomal evasion and sodium sensitivity. 
Although the experimental support for the current model of the pathogen's life cycle was initially obtained by studies of exponential and stationary phase broth cultures, the reciprocal expression of numerous replicative and transmissive traits by $L$. pneumophila has been confirmed by analysing the pathogen's life cycle in macrophages, amoebae and HeLa cells (Table 1). For example, when a stationary phase inoculum of cytotoxic, sodiumsensitive, flagellated and motile cells is incubated with macrophages, the microbes that are ingested subsequently suppress each of these traits during the replication period; as the primary infection period ends with macrophage lysis, the progeny then induce the expression of all four traits (Byrne and Swanson, 1998; Alli et al., 2000).

Likewise, four L. pneumophila proteins known to promote bacterial entry are expressed during the invasion and exit period, but not during the replication phase in macrophages; these proteins are FlaA, Mip, DotO and DotH (Cianciotto and Fields, 1992; Hammer and Swanson, 1999; Dietrich et al., 2001; Watarai et al., 2001a; Wieland et al., 2002). Conversely, the repressor of transmission traits, CsrA, exhibits the opposite pattern of expression: its promoter is active exclusively when intracellular bacteria are multiplying (Molofsky and Swanson, 2003). Remarkably, even the trait that is a hallmark of $L$. pneumophila virulence, lysosome evasion, is inactive during the intracellular replication period (Sturgill-Koszycki and Swanson, 2000; Joshi et al., 2001), as discussed in detail below.

\section{Advantages and limitations of the broth model}

Compared with phagocyte models, synchronous broth cultures offer several technical advantages for studies of L. pneumophila differentiation. With relative ease and economy, large quantities of pure populations of replicative or transmissive bacteria cultured in defined medium can be obtained. Moreover, the samples are free from eukaryotic protein, DNA and RNA. Accordingly, broth cultures are an attractive system for studies of $L$. pneumophila differentiation by modern molecular approaches such as DNA microarrays, proteomics and real-time polymerase chain reaction (PCR). Broth- and agar-grown microbes are also amenable to classical genetic screens, an approach that has already identified several of the activators and repressors of $L$. pneumophila differentiation discussed below.

Although studies of broth-grown microbes have accurately predicted many of the pathogen's behaviours in macrophage or amoebae models (Table 1), more complex experimental systems have revealed additional attributes that are not observed in broth cultures. For example, to replicate to large numbers in macrophages,
L. pneumophila requires an acidic $\mathrm{pH}$, whereas exponential phase broth cultures are acid sensitive (SturgillKoszycki and Swanson, 2000). The bacterial progeny that emerge from amoebae are more infectious than cells harvested from agar (Cirillo et al., 1999). Even more striking is the phenotype of $L$. pneumophila after prolonged culture in HeLa epithelial cells. In this setting, the pathogen differentiates into a thick-walled, pleomorphic, highly resilient and infectious mature intracellular form (MIF) (Faulkner and Garduno, 2002; Garduno et al., 2002), a cell type also observed in amoebae and clinical specimens (Greub and Raoult, 2003). Although this developmental form shares several traits with stationary phase broth-grown L. pneumophila, MIFs appear to be hyperinfectious and -resistant to environmental stress. Analogous to the small cell variant of $C$. burnetii, the spore-like MIF is postulated to be the transmissive form of L. pneumophila in nature (Garduno et al., 2002). Therefore, broth-grown stationary phase microbes probably represent an intermediate stage of a developmental pathway that culminates in the MIF transmissive cell type (Fig. 1). A different habitat in which L. pneumophila survive for extended periods are biofilms, complex microbial communities that pose a significant hazard to potable water supply systems, especially in hospitals (reviewed by Fields et al., 2002). How closely the bacterial forms that develop in either broth or eukaryotic cell culture resemble the cell types that persist in biofilms is a critical open question.

\section{Developmental state determines fate in phagocytes}

To survive ingestion by a phagocyte, L. pneumophila avoids immediate delivery to digestive lysosomes (Horwitz, 1983a,b). This hallmark of the species' virulence dramatically illustrates how microbial differentiation impinges on the host cell response and determines the outcome of the encounter. When transmissive, stationary phase L. pneumophila are phagocytosed, they immediately occupy a spacious cholesterol-rich compartment that does not fuse with lysosomes (Joshi et al., 2001; Watarai et al., 2001b). If L. pneumophila are genetically locked in the transmissive form, the bacteria infect macrophages efficiently, but persist for days without replicating (Molofsky and Swanson, 2003). In contrast, when macrophages are fed $L$. pneumophila that are in the replicative state, the bacteria are delivered to the endocytic network and swiftly killed. In particular, L. pneumophila fail to evade the destructive lysosomes when the transmission regulon is inactive, because the bacteria are in the exponential growth phase, lack an activator of transmission or constitutively express a repressor of transmission (Byrne and Swanson, 1998; Joshi et al., 2001; Molofsky and Swanson, 
2003; regulators depicted in Fig. 2 and described in detail below).

Like exponential phase cells obtained from broth cultures, L. pneumophila that have begun to replicate in macrophages are also delivered to acidic lysosomes. When macrophages are infected with stationary phase $L$. pneumophila, the bacteria persist without multiplying for several hours in a vacuole that appears to be completely separate from endosomal traffic (Horwitz, 1983a,b; Joshi et al., 2001). However, once replication vacuoles contain six or more bacteria, exclusion of the late endosomal and lysosomal protein LAMP-1 is rare (Sturgill-Koszycki and Swanson, 2000). During the next $10-15 \mathrm{~h}$ period, the pathogen replication vacuole continues to accumulate lysosomal markers. Yet, it is evident that the intracellular L. pneumophila are acclimated to this harsh environment, as the bacteria multiply profusely in an acidic vacuole (Sturgill-Koszycki and Swanson, 2000). Thus, transmissive cells pause phagosome maturation, then the bacteria differentiate into an acid-resistant replicative form that exploits phagosome-lysosome fusion to multiply to large numbers.

The model in which L. pneumophila downregulates virulence factors that arrest phagosome maturation during its intracellular replication period is further substantiated by molecular genetic studies of the Dot/lcm type IV secretion system (reviewed by Sexton and Vogel, 2002). The pathogen requires Dot/lcm function to enter cholesterolrich spacious vacuoles, avoid immediate delivery to the endosomal pathway, associate with endoplasmic reticulum and establish its replicative vacuole (Berger et al., 1994; Swanson and Isberg, 1995; Watarai et al., 2001b). Nevertheless, $\operatorname{dot} A$ mutants can replicate to large numbers within macrophages when their initial trafficking defect is bypassed experimentally, either by inducing transient expression of $\operatorname{dot} A$ from a heterologous promoter or by forcing co-habitation in a vacuole with a wild-type transmissive microbe (Roy et al., 1998; Coers et al., 1999). As $\operatorname{dot} A$ is predicted to encode an integral component of the Dot/lcm apparatus (Roy et al., 1998), the data indicate that, although type IV secretion is necessary to block immediate fusion with endosomes, it is dispensable during the period of bacterial replication. Likewise, two other Dot/lcm-dependent traits that promote replication vacuole biogenesis, namely recruitment of vesicles from the endoplasmic reticulum and activation of caspase 3, are vital only during the first 30 min of infection (Kagan and Roy, 2002; Molmeret et al., 2004). In addition to these studies of replication vacuole biogenesis, results of multiple independent genetic and cell biological experiments support the model that the transmissive and replicative states are reciprocal (Table 1). While expressing transmission properties, L. pneumophila do not replicate; conversely, replicating cells do not express transmission traits, including factors that block their delivery to lysosomes.

The host signals that trigger differentiation of transmissive L. pneumophila to the replicative form are not known, but the switch occurs before its delivery to lysosomes. First, vacuoles that harbour as many as four bacterial progeny typically lack the late endosomal/lysosomal protein LAMP-1; not until vacuoles harbour more than five microbes do most contain appreciable LAMP-1. Secondly, when acidification and maturation of phagosomes is inhibited by treating macrophages with the proton pump inhibitor bafilomycin, L. pneumophila multiplication is inhibited, but the bacteria do not arrest at the single-cell stage (Sturgill-Koszycki and Swanson, 2000). Therefore, although an acidic lysosomal network promotes robust microbial growth, a signal other than acidic $\mathrm{pH}$ triggers differentiation to the replicative form. Perhaps after differentiation, during the first cycles of pathogen multiplication, virulence factors that block phagosome maturation become dilute or unstable; consequently, the paused vacuole merges with the lysosomal compartment, a rich source of both nutrients and vacuolar membrane. Alternately, replicative phase L. pneumophila could actively alter gene expression to promote lysosomal fusion.

Like L. pneumophila, the intracellular pathogens $C$. burnetii and certain Leishmania spp. practice a similar 'pregnant pause' strategy to thrive in macrophages. All three pathogens alternate between an infectious stationary phase cell type that initially blocks phagosome maturation and an intracellular form that replicates in acidic lysosomes (reviewed by Swanson and Fernandez-Moreira, 2002). The reciprocal expression of transmission and replication traits is a logical strategy for intracellular pathogens to limit costly energy expenditures. When nutrients are plentiful in the replication niche, transmission phase virulence structures are neither required nor built. Conversely, when conditions are not favourable for growth, the biochemical pathways that promote replication are superfluous and not expressed.

Although the biogenesis of the L. pneumophila replication vacuole has been described in some detail, numerous important questions remain. By what mechanism does $L$. pneumophila arrest phagosome maturation? How are vesicles from the endoplasmic reticulum recruited to the phagosomal membrane? Does the endoplasmic reticulum contribute to bacterial survival? What intracellular cues trigger differentiation of $L$. pneumophila to the replicative form? What is the composition of the vacuole in which the microbe begins to replicate? Knowledge of the regulatory circuitry that controls microbial differentiation can provide experimental tools to investigate the macrophage cell biology that determines the outcome of an L. pneumophila infection.

() 2004 Blackwell Publishing Ltd, Molecular Microbiology, 53, 29-40 


\section{Coupling transmission to the stationary phase}

To escape deteriorating conditions in its replication niche, L. pneumophila co-ordinately activates traits necessary to exit a spent host, survive environmental stress and parasitize its next host, while repressing traits dedicated to intracellular multiplication. Knowledge of the environmental cues and components of the regulatory circuitry that co-ordinate this developmental programme has been obtained from genetic and molecular studies of the $L$. pneumophila life cycle in both broth and phagocyte experimental models.

\section{Alarms}

In broth cultures, L. pneumophila accumulate (p)ppGpp as they exit the exponential growth phase, the amino acid supply is limited, or the expression of relA is induced (Fig. 2; Hammer and Swanson, 1999). Subsequently, the bacteria stop replicating and differentiate into the transmissive form (Fig. 2; Hammer and Swanson, 1999). By analogy with the Escherichia coli stringent response, when L. pneumophila that are replicating in macrophages exhaust the amino acid supply, the enzyme RelA is predicted to be activated. In response to uncharged tRNAs, the ribosome-associated RelA synthase converts GTP to (p)ppGpp. In L. pneumophila broth cultures, this second messenger then co-ordinates entry into stationary phase with expression of traits thought to promote transmission of the progeny to a new host (Fig. 2B; Hammer and Swanson, 1999). Genetic data also support the model in which RelA activity is dedicated to the transmissive phase and is dispensable during the replication period. L. pneumophila relA mutants replicate efficiently inside amoebae and macrophages but, when cultured to stationary phase, they fail to accumulate detectable (p)ppGpp and express some transmission traits poorly, including motility and pigment (Zusman et al., 2002). Nevertheless, the impact of the stringent response on differentiation of intracellular microbes has not been established. For example, (p)ppGpp accumulation during the pathogen's life cycle in phagocytes has not been measured, and the ability of relA mutants to express many of the transmission traits when cultured in either broth or phagocytes has not been examined. The broadly conserved stringent response pathway appears to be monitored by a wide array of pathogens, including Vibrio cholerae, Mycobacterium tuberculosis, Listeria monocytogenes, Streptococcus pyogenes and Pseudomonas aeruginosa (Primm et al., 2000; Chatterji and Kumar Ojha, 2001; Okada et al., 2002; Taylor et al., 2002; Haralalka et al., 2003). By coupling expression of transmission traits to (p)ppGpp accumulation, pathogens can respond to metabolic stress by seeking more fertile territory.

C 2004 Blackwell Publishing Ltd, Molecular Microbiology, 53, 29-40
In addition to the stringent-like response, additional signals and/or regulators appear to control L. pneumophila differentiation both in vitro and in macrophages. For example, the transmission trait defects of relA mutants are milder than those of other regulatory mutants (letA and rpos; discussed below) or replicative microbes obtained from exponential phase cultures of wild-type L. pneumophila (Hammer and Swanson, 1999; Zusman et al., 2002). Accordingly, factors other than RelA probably trigger microbial differentiation. One candidate that has not been examined is the SpoT (p)ppGpp hydrolase/synthase, an enzyme that also generates (p)ppGpp in E. coli and appears to be essential for viability of L. pneumophila (Zusman et al., 2002). Furthermore, bacteria that lack either RelA or the transmission activator LetA appear to spread in macrophage monolayers as efficiently as wild-type microbes, as judged by the rate of increase in cfu throughout a $72 \mathrm{~h}$ infection, a period composed of secondary and tertiary infections (Hammer et al., 2002; Zusman et al., 2002). Therefore, when L. pneumophila are crowded in a replicative vacuole, certain transmission traits may be induced by signal(s) that can bypass both RelA and LetA. As predicted by this model, when compared with their broth counterparts, letA mutants harvested from macrophages are more infectious (B. Byrne and $M$. Swanson, unpublished). Although genome searches suggest that L. pneumophila lacks a classical quorumsensing mechanism, it is plausible that an unorthodox form of quorum sensing induces transmission traits. Alternatively, each replicative L. pneumophila cell may respond independently to other cues in the lysosomal vacuole to activate the transmission programme. As timely differentiation is paramount for intracellular parasites, it is likely that $L$. pneumophila uses multiple redundant pathways to monitor (p)ppGpp levels and other local parameters to judge whether to divide or escape. Because methods to interfere with microbial differentiation could be exploited to prevent or treat infection, identification of alternative signal(s) of differentiation is one imperative of future research.

\section{Sigma factors activate the transmission programme}

To respond to the (p)ppGpp alarmone and differentiate into the transmissive state, $L$. pneumophila requires a number of alternative sigma factors (Fig. 2). The stationary phase factor RpoS $\left(\sigma^{S} / \sigma^{38}\right)$, the flagellar regulator FliA $\left(\sigma^{28}\right)$ and the alternative sigma factor $\operatorname{RpoN}\left(\sigma^{54}\right)$ have been determined genetically to be activators of particular transmission traits (Hales and Shuman, 1999; Bachman and Swanson, 2001; Hammer et al., 2002; Heuner et al., 2002; Heuner and Steinert, 2003; Jacobi et al., 2004). Studies to determine how $L$. pneumophila differentiation 
is co-ordinated by this cohort of alternative sigma factors have been hampered by a lack of knowledge of the effector genes of transmission. Therefore, transcriptional control of the flagellar regulon has been the primary focus of research (for a recent review, see Heuner and Steinert, 2003).

Although the mechanism remains to be examined in $L$. pneumophila, biochemical and genetic studies of $E$. coli by Nystrom and colleagues indicate that ( $p$ )ppGpp acts as a global regulator of transcription by biasing the competition among sigma factors for binding to the RNA core polymerase (Farewell et al., 1998; Jishage et al., 2002; Laurie et al., 2003). In particular, (p)ppGpp appears to destabilize the interaction of the predominant vegetative sigma factor, $\sigma^{\mathrm{D}}\left(\sigma^{70}\right)$, with RNA core polymerase. As a consequence, an alternative sigma factor can replace $\sigma^{\mathrm{D}}$ in the core and recruit the enzyme to its cohort of promoters. Accordingly, ( $p$ )ppGpp accumulation is predicted to increase the amount of RpoS protein and also the activity of both RpoS and RpoN (Jishage et al., 2002). The prediction that L. pneumophila RpoS competes with other sigma factors for binding to RNA core polymerase is consistent with the phenotype of bacteria that lack or overexpress rpoS. For example, when present in multiple copies, rpoS inhibits the expression of three fliA-dependent transmission traits in primary murine macrophages (Bachman and Swanson, 2004a) and inhibits intracellular growth in Acanthamoeba castellanii (Hales and Shuman, 1999). How (p)ppGpp couples expression of RpoS-dependent stationary phase traits with FliA-dependent transmission traits can be analysed biochemically once genes that encode effectors of the L. pneumophila transmissive state are identified.

Legionella pneumophila requires the flagellar sigma factor FliA not only to synthesize the flagellar filament and for motility (Fettes et al., 2001; Heuner et al., 2002), but also to express contact-dependent cytotoxicity, lysosome evasion in macrophages and replication in the social amoebae Dictyostelium discoideum (Hammer et al., 2002; Heuner et al., 2002; L. M. Shetron-Rama and M. S. Swanson, unpublished). Therefore, the FliA sigma factor $\left(\sigma^{28}\right)$ of $L$. pneumophila may activate promoters of the flagellar regulon as well as other virulence effector genes (Hammer et al., 2002; Heuner et al., 2002; Molofsky and Swanson, 2003), a pattern also observed in Salmonella enterica (Eichelberg and Galan, 2000). An alternative mechanism that links motility to the expression of additional virulence traits is illustrated by Vibrio cholerae, which responds to flagellar motion and sodium gradients in a complex manner to alter expression of the ToxT virulence regulon (Hase and Mekalanos, 1999). Whatever the mechanism, identification of the cohort of genes regulated by FliA together with analysis of representative flagellar development mutants can provide insight to how L. pneu- mophila escapes from one host, then blocks phagosome maturation in the next.

\section{Post-transcriptional repression of the transmission regulon}

Whereas the alternative sigma factors RpoS, FliA and RpoN govern transcription initiation to induce transmission traits, post-transcriptional regulation of this class of mRNAs is likely to be controlled by the two-component regulatory system LetA/S. Originally identified in an $L$. pneumophila screen for mutants defective for flagellar synthesis, LetA/S, together with the novel protein LetE, induces a large panel of transmissive traits in response to the alarmone (p)ppGpp (Hammer et al., 2002; Lynch et al., 2003; Bachman and Swanson, 2004b). Several diverse bacterial species also rely on homologues of LetA/ S, called ExpA/S, GacA/S, UvrY/BarA, VarA/S and SirA/ BarA, to express a variety of extracellular virulence factors and to modulate carbon pathways when conditions deteriorate (reviewed by Heeb and Haas, 2001). Whether (p)ppGpp activates the membrane-bound sensor kinase LetS is not known but, in homologous systems, an active LetS sensor kinase phosphorylates the LetA response regulator to change gene expression. Consistent with the regulatory hierarchies delineated in $E$. coli and other microbes, genetic data indicate that the major, if not sole, function of activated LetA is to relieve repression by the global regulatory RNA-binding protein CsrA (RsmA).

CsrA belongs to a highly conserved family of global regulators that typically control stationary phase traits post-transcriptionally (reviewed by Romeo, 1998). In E. coli, CsrA binds near the ribosomal binding site of the $g l g C$ and $c s t A$ mRNA transcripts, preventing their translation and promoting premature degradation (Liu and Romeo, 1997; Dubey et al., 2003). CsrA can also stabilize transcripts, including those of the master flagellar regulatory locus flhDC (Wei et al., 2001). Microarray analysis revealed that the CsrA of Salmonella enterica co-ordinately controls a host of metabolic pathways as well as virulence traits encoded by the SPI-1 pathogenicity island (Lawhon et al., 2003). In E. coli, CsrA activity is inhibited when the repressor is bound by either of the two untranslated regulatory RNAs known as $\operatorname{csr} B$ and $c s r C$, which are induced by UvrY/BarA (LetA/S homologues). In L. pneumophila, every transmission trait that has been examined is repressed by CsrA (Table 1, Fig. 2B). Moreover, genetic inactivation of the repressor csrA bypasses the requirement for the let $A$ inducer of the transmission phenotype (Fig. 2B; Molofsky and Swanson, 2003). Accordingly, by analogy with other Gram-negative bacteria, it is likely that LetA/S in L. pneumophila induces as yet unidentified csrBlike regulatory $\mathrm{RNA}(\mathrm{s})$ that bind and inhibit CsrA activity when nutrients are limiting, thereby inducing virulence 
traits and perhaps metabolic pathways that promote transmission of L. pneumophila.

A subset of the CsrA-repressed traits requires the flagellar sigma factor FliA for its transcription (Fig. 2B). Specifically, CsrA represses and FliA activates the transmissive phase traits of motility: contact-dependent cytotoxicity and immediate evasion of lysosomes (Table 1; Fettes et al., 2001; Hammer et al., 2002; Heuner et al., 2002; Molofsky and Swanson, 2003). Accordingly, CsrA is predicted to inhibit either fliA mRNA stability or translation directly or fliA message production indirectly. An efficient mechanism for CsrA to control multiple transmissive traits would be to repress the master switch of the flagellar regulon in L. pneumophila, presumably RpoN and/or FleQ (Fettes et al., 2001; Heuner and Steinert, 2003; Jacobi et al., 2004), but this possibility has not been examined. As CsrA represses multiple phenotypes linked to $L$. pneumophila virulence, identification of the mRNA species that it targets is a viable approach to delineating the molecular mechanisms of $L$. pneumophila pathogenesis.

\section{Dot/lcm regulation}

The type IV secretion system remains the best characterized virulence factor of $L$. pneumophila; accordingly, its substrates and transcriptional regulation have been the focus of considerable investigative effort. Encoded by the defective in organelle transport/intracellular multiplication loci of $L$. pneumophila, the apparatus is postulated to secrete the virulence effectors that immediately isolate the pathogen vacuole from the endocytic pathway (reviewed by Sexton and Vogel, 2002). In addition to its effects on macrophage cell biology, the Dot/lcm complex translocates protein-plasmid DNA complexes between bacteria (Vogel et al., 1998).

Whether L. pneumophila express a functional type IV secretion apparatus in both the replicative and transmissive phase has not been established definitively. The efficiency of plasmid transfer by pure cultures of exponential and stationary phase $L$. pneumophila has not been reported, although it appears that bacteria replicating on solid agar fail to conjugate plasmid DNA (Segal and Shuman, 1998). A series of studies using lac $Z$ translational fusions demonstrated that, of nine dot/icm loci examined, the products of five were more abundant in the stationary phase of broth cultures but, in each case, the effect was modest (Gal-Mor et al., 2002). Of these, only icmP expression was reduced by mutation of relA, rpoS or letA (Zusman et al., 2002; Gal-Mor and Segal, 2003a). Whether the two- to threefold increase in expression of a subset of the dot/icm genes that occurs when L. pneumophila enter the stationary phase in broth contributes to the dramatic concomitant increase in lysosome evasion has not been tested. Also, the effect of LetA on transcription and translation of particular dot/icm genes warrants more detailed study, as another group who analysed total RNA instead of reporter constructs concluded that LetA is a strong inducer of $\operatorname{dotA}$ expression (Lynch et al., 2003). A second activator, the periplasmic stress two-component response regulator $\mathrm{CpxR}$, strongly induces expression of $i c m R$, a non-structural gene of the secretion apparatus, and more modestly activates the $\operatorname{dotA} / \mathrm{icm} V$ and icmW/X operons in broth cultures (Gal-Mor and Segal, 2003b). However, as $c p x R$ mutants replicate efficiently in macrophages and amoebae (Gal-Mor and Segal, 2003b), L. pneumophila must encode another mechanism to generate sufficient $d o t /$ icm expression to establish its replication niche. Biochemical studies of the Dot/lcm complex throughout the life cycle in broth and phagocyte culture are needed to determine whether regulated synthesis or assembly of the secretion system accounts for the dramatically different intracellular fates of replicative and transmissive L. pneumophila.

One possibility is that the type IV secretion apparatus is synthesized constitutively, but the effector substrates are expressed solely during the transmission phase. Proteins likely to be translocated by the Dot/lcm system have been identified by a variety of genetic strategies, and the expression pattern of the proteins and the ability of the corresponding mutants to establish replication vacuoles has been examined. As predicted for virulence factors that govern phagosome maturation, the RalF, LidA and SidC proteins are each induced when $L$. pneumophila is cultured to the stationary phase in broth. Furthermore, when cultured in macrophages, all three proteins are translocated to the phagosomal membrane by a $\operatorname{dot} A$-dependent process (Nagai and Roy, 2001; Nagai et al., 2002; Conover et al., 2003; Luo and Isberg, 2004). Nevertheless, none of these translocated proteins is required by the pathogen either to evade the endosomal network or to replicate in macrophages. Either the RalF, LidA and SidC proteins are redundant or they contribute to some other aspect of the pathogen's life cycle. The model of the genetic circuitry that controls the L. pneumophila life cycle (Fig. 2) can provide a conceptual framework for the design of experimental strategies to identify virulence factors that directly alter membrane traffic in phagocytes, which are predicted to be targets of FliA, CsrA and RpoS regulation.

\section{Impact of differentiation on experimental design}

Appreciation that the expression of transmission and replication traits by intracellular pathogens is probably reciprocal and also genetically separable can facilitate the design and interpretation of classical and molecular genetic analyses to identify their virulence mechanisms. Typically, experimentalists focus on those virulence determinants that have a measurable impact on replication, as 
judged by the slope of intracellular growth curve plots. Given the reciprocal pattern of the transmission and replication phases, this strategy risks discounting determinants that promote immediate bacterial adherence, entry and lysosome evasion, traits that are likely to be vital to the establishment of infection in human hosts. For example, L. pneumophila that lack the LetA/S or FliA positive activators, the Hsp60 or RtxA surface proteins or the FlaA flagellar filament protein have discernable phenotypes when initial host-pathogen interactions are quantified. Nevertheless, the minority of each of the mutant microbes that adhere and survive phagocytosis proceeds to replicate at wild-type rates (Garduno et al., 1998; Cirillo et al., 2001; Dietrich et al., 2001; Hammer et al., 2002; Heuner et al., 2002; Lynch et al., 2003). Therefore, unless early events are quantified, the contribution of this class of transmission factors to L. pneumophila pathogenesis cannot be appreciated. On the other hand, certain transmission defects do prevent subsequent intracellular growth: stationary phase $\operatorname{dot} A$ and $\operatorname{dot} B$ mutants of $L$. pneumophila fail to establish replication vacuoles and, instead, persist without replicating in a non-toxic endosomal compartment (Joshi et al., 2001). By designing specific assays to measure either transmission or replication, both classes of virulence factors can be identified.

In the post-genomic era, a comprehensive analysis of microbial adaptation to a particular environment can be achieved by applying powerful molecular genetic methods such as microarrays, proteomics, in vitro expression technology and signature-tagged mutagenesis. As L. pneumophila differentiates in response to nutrient levels, insight into the physiological pathways that distinguish its transmissive and replicative stages can be obtained by comparing synchronous exponential and stationary phase populations obtained from chemically defined medium. In contrast, the heterogeneity of cell populations harvested from agar will obscure the microbe's biphasic design. Whether candidate loci are indeed dedicated to one phase of the pathogen's life cycle can then be confirmed by more laborious analysis of phagocyte cultures. By incorporating knowledge of microbial differentiation into experimental design, the physiological patterns obtained by modern genomic methods can be brought into sharp focus to illuminate the mechanisms of microbial pathogenesis.

\section{Acknowledgements}

We thank Drs Brian Hammer and Mike Bachman for their seminal contributions to the paradigm presented here, the members of the Swanson laboratory for many thoughtful conversations, and Dr Klaus Heuner for sharing over the past several years his insights into L. pneumophila virulence regulation. Also thanks to Dr Gil Segal for his critical discussions.
This work was supported by the NIH National Research Service Award no. 5-T32-GM07544 from the National Institute of General Medicine Sciences, NIH grant Al 44212-01 and the University of Michigan Medical Scientist Training Program.

\section{References}

Alli, O.A., Gao, L.Y., Pedersen, L.L., Zink, S., Radulic, M., Doric, M., and Abu Kwaik, Y. (2000) Temporal pore formation-mediated egress from macrophages and alveolar epithelial cells by Legionella pneumophila. Infect Immun 68: 6431-6440.

Bachman, M.A., and Swanson, M.S. (2001) RpoS co-operates with other factors to induce Legionella pneumophila virulence in the stationary phase. Mol Microbiol 40: 12011214.

Bachman, M.A., and Swanson, M.S. (2004a) Genetic evidence that Legionella pneumophila RpoS modulates expression of the transmission phenotype in both the exponential phase and the stationary phase. Infect Immun 72: 2468-2476.

Bachman, M.A., and Swanson, M.S. (2004b) The LetE protein enhances expression of multiple LetA/LetSdependent transmission traits by Legionella pneumophilia. Infect Immun 72(6).

Berger, K.H., Merriam, J.J., and Isberg, R.I. (1994) Altered intracellular targeting properties associated with mutations in the Legionella pneumophila dotA gene. Mol Microbiol 14: 809-822.

Byrne, B., and Swanson, M.S. (1998) Expression of Legionella pneumophila virulence traits in response to growth conditions. Infect Immun 66: 3029-3034.

Chatterji, D., and Kumar Ojha, A. (2001) Revisiting the stringent response, ppGpp and starvation signaling. Curr Opin Microbiol 4: 160-165.

Cianciotto, N.P., and Fields, B.S. (1992) Legionella pneumophila mip gene potentiates intracellular infection of protozoa and human macrophages. Proc Natl Acad Sci USA 89: 5188-5191.

Cirillo, J.D., Cirillo, S.L., Yan, L., Bermudez, L.E., Falkow, S., and Tompkins, L.S. (1999) Intracellular growth in Acanthamoeba castellanii affects monocyte entry mechanisms and enhances virulence of Legionella pneumophila. Infect Immun 67: 4427-4434.

Cirillo, S.L., Bermudez, L.E., El-Etr, S.H., Duhamel, G.E., and Cirillo, J.D. (2001) Legionella pneumophila entry gene rtxA is involved in virulence. Infect Immun 69: 508517.

Coers, J., Monahan, C., and Roy, C.R. (1999) Modulation of phagosome biogenesis by Legionella pneumophila creates an organelle permissive for intracellular growth. Nature Cell Biol 1: 451-453.

Conover, G.M., Derre, I., Vogel, J.P., and Isberg, R.R. (2003) The Legionella pneumophila LidA protein: a translocated substrate of the Dot/lcm system associated with maintenance of bacterial integrity. Mol Microbiol 48: 305321.

Cotter, P.A., and Jones, A.M. (2003) Phosphorelay control of virulence gene expression in Bordetella. Trends Microbiol 11: 367-373.

Dietrich, C., Heuner, K., Brand, B.C., Hacker, J., and Stein- 
ert, M. (2001) Flagellum of Legionella pneumophila positively affects the early phase of infection of eukaryotic host cells. Infect Immun 69: 2116-2122.

Dubey, A.K., Baker, C.S., Suzuki, K., Jones, A.D., Pandit, P., Romeo, T., and Babitzke, P. (2003) CsrA regulates translation of the Escherichia coli carbon starvation gene, cstA, by blocking ribosome access to the cstA transcript. $J$ Bacteriol 185: 4450-4460.

Eichelberg, K., and Galan, J.E. (2000) The flagellar sigma factor FliA (sigma (28) regulates the expression of Salmonella genes associated with the centisome 63 type III secretion system. Infect Immun 68: 2735-2743.

Farewell, A., Kvint, K., and Nystrom, T. (1998) Negative regulation by RpoS: a case of sigma factor competition. Mol Microbiol 29: 1039-1051.

Faulkner, G., and Garduno, R.A. (2002) Ultrastructural analysis of differentiation in Legionella pneumophila. J Bacteriol 184: 7025-7041.

Fettes, P.S., Forsbach-Birk, V., Lynch, D., and Marre, R. (2001) Overexpresssion of a Legionella pneumophila homologue of the $E$. coli regulator csrA affects cell size, flagellation, and pigmentation. Int $J$ Med Microbiol 291: 353-360.

Fields, B.S., Benson, R.F., and Besser, R.E. (2002) Legionella and Legionnaires' disease: 25 years of investigation. Clin Microbiol Rev 15: 506-526.

Gal-Mor, O., and Segal, G. (2003a) The Legionella pneumophila GacA homolog (LetA) is involved in the regulation of icm virulence genes and is required for intracellular multiplication in Acanthamoeba castellanii. Microb Pathog 34: 187-194.

Gal-Mor, O., and Segal, G. (2003b) Identification of CpxR as a positive regulator of icm and dot virulence genes of Legionella pneumophila. J Bacteriol 185: 4908-4919.

Gal-Mor, O., Zusman, T., and Segal, G. (2002) Analysis of DNA regulatory elements required for expression of the Legionella pneumophila icm and dot virulence genes. $J$ Bacteriol 184: 3823-3833.

Garduno, R.A., Garduno, E., and Hoffman, P.S. (1998) Surface-associated hsp60 chaperonin of Legionella pneumophila mediates invasion in a HeLa cell model. Infect Immun 66: 4602-4610.

Garduno, R.A., Garduno, E., Hiltz, M., and Hoffman, P.S. (2002) Intracellular growth of Legionella pneumophila gives rise to a differentiated form dissimilar to stationaryphase forms. Infect Immun 70: 6273-6283.

Greub, G., and Raoult, D. (2003) Morphology of Legionella pneumophila according to their location within Hartmanella vermiformis. Res Microbiol 154: 619-621.

Hales, L.M., and Shuman, H.A. (1999) The Legionella pneumophila rpos gene is required for growth within Acanthamoeba castellanii. J Bacteriol 181: 4879-4889.

Hammer, B.K., and Swanson, M.S. (1999) Co-ordination of Legionella pneumophila virulence with entry into stationary phase by ppGpp. Mol Microbiol 33: 721-731.

Hammer, B.K., Tateda, E.S., and Swanson, M.S. (2002) A two-component regulator induces the transmission phenotype of stationary-phase Legionella pneumophila. Mol Microbiol 44: 107-118.

Hammerschlag, M.R. (2002) The intracellular life of chlamydiae. Semin Pediatr Infect Dis 13: 239-248.
Haralalka, S., Nandi, S., and Bhadra, R.K. (2003) Mutation in the relA gene of Vibrio cholerae affects in vitro and in vivo expression of virulence factors. J Bacteriol 185: 46724682.

Hase, C.C., and Mekalanos, J.J. (1999) Effects of changes in membrane sodium flux on virulence gene expression in Vibrio cholerae. Proc Natl Acad Sci USA 96: 3183-3187.

Heeb, S., and Haas, D. (2001) Regulatory roles of the GacS/ GacA two-component system in plant-associated and other gram-negative bacteria. Mol Plant-Microbe Interact 14: 1351-1363.

Heuner, K., and Steinert, M. (2003) The flagellum of Legionella pneumophila and its link to the expression of the virulent phenotype. Int J Med Microbiol 293: 133-143.

Heuner, K., Brand, B.C., and Hacker, J. (1999) The expression of the flagellum of Legionella pneumophila is modulated by different environmental factors. FEMS Microbiol Lett 175: 69-77.

Heuner, K., Dietrich, C., Steinert, M., Gobel, U.B., and Hacker, J. (2000) Cloning and characterization of a Legionella pneumophila-specific gene encoding a member of the LysR family of transcriptional regulators. Mol Gen Genet 264: 204-211.

Heuner, K., Dietrich, C., Skriwan, C., Steinert, M., and Hacker, J. (2002) Influence of the alternative sigma (28) factor on virulence and flagellum expression of Legionella pneumophila. Infect Immun 70: 1604-1608.

Hiltz, M.F., Sisson, G.R., Brassinga, A.K.C., Garduno, E., Garduno, R.A., and Hoffman, P.S. (2004) Expression of magA in Legionella pneumophila Philadelphia-1 is developmentally regulated and a marker of formation of mature intracellular forms. J Bacteriol 186(10).

Horwitz, M.A. (1983a) The Legionnaires' disease bacterium (Legionella pneumophila) inhibits phagosome-lysosome fusion in human monocytes. J Exp Med 158: 2108-2126.

Horwitz, M.A. (1983b) Formation of a novel phagosome by the Legionnaires' disease bacterium (Legionella pneumophila) in human monocytes. J Exp Med 158: 1319-1331.

Jacobi, S., Schade, R., and Heuner, K. (2004) Characterization of the alternative sigma factor (54) and the transcriptional regulator FleQ of Legionella pneumophila, which are both involved in the regulation cascade of flagellar gene expression. J Bacteriol 186: 2540-2547.

Jishage, M., Kvint, K., Shingler, V., and Nystrom, T. (2002) Regulation of sigma factor competition by the alarmone ppGpp. Genes Dev 16: 1260-1270.

Joshi, A.D., Sturgill-Koszycki, S., and Swanson, M.S. (2001) Evidence that Dot-dependent and - independent factors isolate the Legionella pneumophila phagosome from the endocytic network in mouse macrophages. Cell Microbiol 3: 99-114.

Kagan, J.C., and Roy, C.R. (2002) Legionella phagosomes intercept vesicular traffic from endoplasmic reticulum exit sites. Nature Cell Biol 4: 945-954.

Laurie, A.D., Bernardo, L.M., Sze, C.C., Skarfstad, E., Szalewska-Palasz, A., Nystrom, T., and Shingler, V. (2003) The role of the alarmone (p)ppGpp in sigma $N$ competition for core RNA polymerase. $J$ Biol Chem 278: 1494-1503.

Lawhon, S.D., Frye, J.G., Suyemoto, M., Porwollik, S., McClelland, M., and Altier, C. (2003) Global regulation by 
CsrA in Salmonella typhimurium. Mol Microbiol 48: 16331645.

Liu, M.Y., and Romeo, T. (1997) The global regulator CsrA of Escherichia coli is a specific mRNA-binding protein. $J$ Bacteriol 179: 4639-4642.

Luo, Z.Q., and Isberg, R.R. (2004) Multiple substrates of the Legionella pneumophila Dot/lcm system identified by interbacterial protein transfer. Proc Natl Acad Sci USA 101: 841-846.

Lynch, D., Fieser, N., Gloggler, K., Forsbach-Birk, V., and Marre, R. (2003) The response regulator LetA regulates the stationary-phase stress response in Legionella pneumophila and is required for efficient infection of Acanthamoeba castellanii. FEMS Microbiol Lett 219: 241248.

Molmeret, M., Zink, S.D., Han, L., Abu-Zant, A., Asari, R., Bitar, D.M., and Abu Kwaik, Y. (2004) Activation of caspase-3 by the Dot/lcm virulence system is essential for arrested biogenesis of the Legionella-containing phagosome. Cell Microbiol 6: 33-48.

Molofsky, A.B., and Swanson, M.S. (2003) Legionella pneumophila CsrA is a pivotal repressor of transmission traits and activator of replication. Mol Microbiol 50: 445-461.

Nagai, H., and Roy, C.R. (2001) The DotA protein from Legionella pneumophila is secreted by a novel process that requires the Dot/lcm transporter. EMBO J 20: 59625970.

Nagai, H., Kagan, J.C., Zhu, X., Kahn, R.A., and Roy, C.R. (2002) A bacterial guanine nucleotide exchange factor activates ARF on Legionella phagosomes. Science 295: 679682.

Okada, Y., Makino, S., Tobe, T., Okada, N., and Yamazaki, S. (2002) Cloning of rel from Listeria monocytogenes as an osmotolerance involvement gene. Appl Environ Microbiol 68: 1541-1547.

Primm, T.P., Andersen, S.J., Mizrahi, V., Avarbock, D., Rubin, H., and Barry, C.E., III (2000) The stringent response of Mycobacterium tuberculosis is required for long-term survival. J Bacteriol 182: 4889-4898.

Romeo, T. (1998) Global regulation by the small RNA-binding protein CsrA and the non-coding RNA molecule CsrB. Mol Microbiol 29: 1321-1330.

Rowbotham, T.J. (1986) Current views on the relationships between amoebae, legionellae and man. Isr J Med Sci 22: 678-689.

Roy, C.R., Berger, K.H., and Isberg, R.R. (1998) Legionella pneumophila DotA protein is required for early phagosome trafficking decisions that occur within minutes of bacterial uptake. Mol Microbiol 28: 663-674.

Samuel, J.E., Kiss, K., and Varghees, S. (2003) Molecular pathogenesis of Coxiella burnetii in a genomics era. Ann NY Acad Sci 990: 653-663.

Segal, G., and Shuman, H.A. (1998) Intracellular multiplication and human macrophage killing by Legionella pneumophila are inhibited by conjugal components of IncQ plasmid RSF1010. Mol Microbiol 30: 197-208.

Sexton, J.A., and Vogel, J.P. (2002) Type IVB secretion by intracellular pathogens. Traffic 3: 178-185.

Sturgill-Koszycki, S., and Swanson, M.S. (2000) Legionella pneumophila replication vacuoles mature into acidic, endocytic organelles. J Exp Med 192: 1261-1272.

Swanson, M., and Fernandez-Moreira, E. (2002) A microbial strategy to multiply in macrophages: the pregnant pause. Traffic 3: 170-177.

Swanson, M.S., and Hammer, B.K. (2000) Legionella pneumophila pathogenesis: a fateful journey from amoebae to macrophages. Annu Rev Microbiol 54: 567-613.

Swanson, M.S., and Isberg, R.R. (1995) Association of Legionella pneumophila with the macrophage endoplasmic reticulum. Infect Immun 63: 3609-3620.

Taylor, C.M., Beresford, M., Epton, H.A., Sigee, D.C., Shama, G., Andrew, P.W., and Roberts, I.S. (2002) Listeria monocytogenes relA and hpt mutants are impaired in surfaceattached growth and virulence. J Bacteriol 184: 621-628.

Vogel, J.P., Andrews, H.L., Wong, S.K., and Isberg, R.R. (1998) Conjugative transfer by the virulence system of Legionella pneumophila. Science 279: 873-876.

Watarai, M., Andrews, H.L., and Isberg, R.R. (2001a) Formation of a fibrous structure on the surface of Legionella pneumophila associated with exposure of DotH and DotO proteins after intracellular growth. Mol Microbiol 39: 313329.

Watarai, M., Derre, I., Kirby, J., Growney, J.D., Dietrich, W.F., and Isberg, R.R. (2001b) Legionella pneumophila is internalized by a macropinocytotic uptake pathway controlled by the Dot/lcm system and the mouse Lgn1 locus. J Exp Med 194: 1081-1096.

Wei, B.L., Brun-Zinkernagel, A.M., Simecka, J.W., Pruss, B.M., Babitzke, P., and Romeo, T. (2001) Positive regulation of motility and flhDC expression by the RNA-binding protein CsrA of Escherichia coli. Mol Microbiol 40: 245256.

Wieland, H., Faigle, M., Lang, F., Northoff, H., and Neumeister, B. (2002) Regulation of the Legionella mip-promoter during infection of human monocytes. FEMS Microbiol Lett 212: 127-132.

Zusman, T., Gal-Mor, O., and Segal, G. (2002) Characterization of a Legionella pneumophila relA insertion mutant and roles of RelA and RpoS in virulence gene expression. $J$ Bacteriol 184: 67-75. 